\title{
Lobar infiltration by squamous cell carcinoma
}

\section{R K HIND}

From the Department of Respiratory Medicine, Hammersmith Hospital, London

Lobar consolidation without collapse is caused by infection distal to bronchial obstruction in the vast majcrity of cases of squamous cell carcinoma of the lung. ${ }^{1}$ In this report the histological and radiological behaviour of a squamous cell carcinoma closely resembled that of an alveolar cell carcinoma.

\section{Case report}

A 74-year-old retired bricklayer had been followed by the respiratory department since 1963 for chronic bronchitis. When first seen his forced expiratory volume in one second was 0.631 , and vital capacity 1.251 , with a slight bronchodilator response, and a normal mixed venous carbon dioxide and transfer factor for carbon monoxide. A subsequent steroid trial was negative. He had smoked 30 cigarettes a day between 1920 and 1963, when he stopped completely. Over the next 16 years the natural history of his disease was closely followed. He had three acute exacerbations requiring inpatient treatment botween 1974 and 1979, developed carbon dioxide retention in 1975, and cor pulmonale in 1978 .

In April 1978 abdominoperineal excision of the rectum was performed for a Duke's stage A welldifferentiated adenocarcinoma; postoperative recovery was uneventful.

In April 1979, the patient was readmitted to hospital with a two-week history of increasing cough, productive of foul green sputum, increasing dyspnoea, and ankle oedema. Two days previously amoxycillin, $250 \mathrm{mg}$ eight-hourly, had teen started. On examination, there was pyrexia, central and peripheral cyanosis, and evidence of congestive cardiac failure. Dullness to percussion and reduced breath sounds were present over the left upper and mid zones posteriorly.

Clinical diagnoses of cardiac and respiratory failure, secondary to an acute infective exacerbation of chronic bronchitis were made. Investigations showed a leucocytosis of $13.8 \times 10^{9} 1^{-1}$, with $93 \%$ neutrophils, and diffuse shadowing in the left upper lobe on the chest radiograph. Sputum and blood cultures were negative. Blood gas analysis confirmed the presence of respiratory failure. The $\mathrm{FEV}_{1}$ was 0.51 , and VC 1.101 .

Treatment was started with controlled oxygen, diuretics, bronchodilators, physiotherapy, and the

Address for reprint requests: Dr CRK Hind, The National Hosp:tal for Nervous Diseases, Queen Square, London WC1N 3BG. amoxycillin was continued. Over the next four days he made a gradual improvement.

On day 5 , one litre of blood-stained pus drained spontaneously from the site of the previous perineal surgery. $E$ coli was grown on culture, which was resistant to ampicillin, but sensitive to cotrimoxazole. No malignant cells were seen on cytological examination. A course of cotrimoxazole was started. The perineal wound healed uneventfully. Three days after completing a 10-day course of cotrimoxazole, the patient's condition deteriorated. Further symptoms and signs of a chest infection developed, and sputum culture grew $\boldsymbol{H}$ influenzae, resistant to ampicillin and sulphonanides, but sensitive to erythromycin. A repeat radiograph showed further increase in the diffuse shadowing in the left upper lobe, now mainly in the lingula and posterior segment, with no evidence of collapse. A course of erythomycin was started, and the patient's condition improved. Six days later, however, a repeat sputum culture revealed $H$ influenzae only marginally sensitive to erythromycin. Another course of cotrimoxazole was given.

Despite a slight improvement in the clinical state, the radiographic appearances did not improve, with persistent consolidation in the lingula and posterior segment of the left upper lobe and no evidence of collapse (fig 1). Repeat bacteriological cultures of blood, and sputum (for aerobes, anaerobes, fungi, Nocardia, and mycobacteria) were negative, as were repeated examinations of the sputum for malignant cells. Viral antibodies and cold agglutinins were

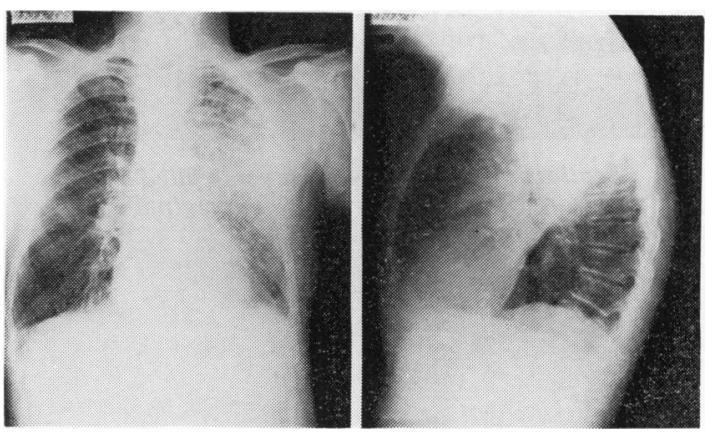

Fig $1 P A$ and left lateral chest radiograph, showing consolidation in the posterior segment of the left upper lobe and lingula. 
negative. Tomography of the left hilar region showed no evidence of obstruction. Heaf testing showed a grade II response. All haematological and biochemical tests were normal.

Rigid and fibreoptic bronchoscopy were normal apart from minor inflammatory changes in the left upper lobe bronchi. No biopsies were taken. Bacteriological and cytological examination of the bronchial aspirates were negative.

After bronchoscopy the patient was started on metronidazole on an empirical basis. The radiographic shadowing persisted, and the patient died in June.

\section{Necropsy findings}

There was local recurrence of a well-differentiated adenocarcinoma in the pelvis, but no evidence of distant spread. The lingula and apical and posterior segments of the left upper lobe of the lung were diffusely infiltrated by tumour. Histologically, this was a moderately differentiated squamous cell carcinoma. No major bronchi or bronchioles were occluded, but there was diffuse intra-alveolar and peribronchiolar spread of the tumour (fig 2). The hilar nodes were replaced by tumour, and squamous cell metastases were present in the liver. The remainder of the lung showed changes compatible with chronic bronchitis and centriacinar emphysema.

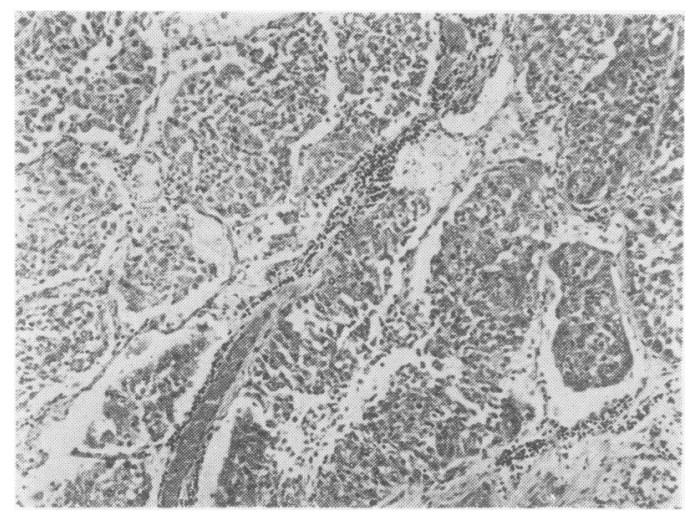

Fig 2 Section of lung showing alveoli filled with squamous carcinoma cells. $H$ and $E$, original magnification $\times 150$.

\section{Discussion}

Squamous cell carcinomas account for between 37.85 and $61.2 \%$ of primary lung tumours. The vaspe majority arise in the large bronchi, and present with symptoms and signs of bronchial obstruction. The spread mainly in the extracartilaginous parts of the bronchial wall invading the adjacent lymph nodes directly, and are particularly liable to undergo cen-s tral necrosis. ${ }^{2}$

Radiologically, the appearances are of atelectasi $(37 \%)$, a hilar or perihilar mass $(35 \%)$ or of a obstructive pneumonitis or consolidation $(25 \%)$ Analysis of the radiographic appearances of $26 \%$ cases of squamous cell carcinoma of the lung b\% Byrd and others, ${ }^{3}$ revealed only two cases with homogeneous opacification of a lobe or segments without decrease in volume. No clinical or patho? logical details of these two cases were given.

This radiological appearance of lobar consolidar tion not caused by proximal obstruction is the usual finding in cases of alveolar cell carcinomasos with invasion of a bronchiole and local spread of the cells into the alveoli which are then lined bog them. ${ }^{4}$

Therefore, on histological and radiographif grounds, this squamous cell carcinoma behaved i an atypical manner, following the pattern of as alveolar cell carcinoma.

I thank Dr JMB Hughes for his help and advice and for permission to report this case, Prof essor Azzopardi for advice, and Denise Brinkman for he secretarial help.

\section{References}

1 Byrd RB, Carr DT, Miller WE, Payne WSA Woolner LB. Radiographic abnormalities in car cinoma of the lung as related to histological ce type. Thorax 1969; 24:573-5.

2 Spencer H. Carcinoma of the lung. In: Patholog of the lung. Third edition. Oxford: Pergamo Press, 1977: 773-81.

3 Byrd RB, Miller WE, Carr DT, Payne wS Woolner LB. The roentgenographic appearanco of squamous cell carcinoma of the bronchus? Mayo Clin Proc 1968; 43:327-32.

4 Simon G. Homogeneous shadows groupe according to shape, size and distribution. In Principles of chest $x$-ray diagnosis. Fourt edition. London: Butterworths, 1978: 52-62. 